\title{
Impact of COVID-19 lockdown measures on a cohort of eating disorders patients
}

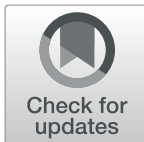

Paulo P. P. Machado ${ }^{*^{*} \mathbb{D}}$, Ana Pinto-Bastos ${ }^{1} \mathbb{D}$, Rita Ramos', Tânia F. Rodrigues ${ }^{1} \mathbb{D}$, Elsa Louro ${ }^{1}$, Sónia Gonçalves ${ }^{1} \mathbb{D}$, Isabel Brandão ${ }^{2}$ and Ana Vaz ${ }^{1}$ (D)

\begin{abstract}
Background: Lockdown implemented to prevent the COVID-19 spread resulted in marked changes in the lifestyle. The objective of the current study was to assess the impact of lockdown measures on a cohort of eating disorder (ED) patients being followed as part of an ongoing naturalistic treatment study.

Methods: Ninety-nine patients aged 18 or older, currently or previously, in treatment at a Portuguese specialized hospital unit were contacted by phone and invited to participate in the current survey. Fifty-nine agreed to be interviewed by phone, and 43 agreed to respond to a set of self-report measures of ED symptoms, emotion regulation difficulties, clinical impairment, negative urgency, and COVID-19 impact, during the week after the end of the lockdown period.

Results: Data showed that of the 26 patients currently in treatment: 8 remained unchanged (31\%), 7 deteriorated (27\%), and 11 reliably improved (42\%). Of the 17 participants not currently in treatment: 3 deteriorated (18\%), 9 remained unchanged (53\%), and 5 (29\%) improved after the lockdown period. The Coronavirus Impact Scale showed that most patients considered their routines moderately or extremely impacted, experienced stress related to coronavirus, and showed difficulty in maintaining physical exercise and feeding routines. Results suggest that higher impact of COVID-19 lockdown was significantly correlated with eating disorder symptoms and associated psychopathology, impulsivity, difficulties in emotion regulation and clinical impairment measured at post-lockdown. In addition, the impact of COVID-19 and lockdown measures on clinical impairment was mediated by difficulties in emotion regulation.
\end{abstract}

Conclusions: Findings suggest that some ED patients may experience worsening of their condition, especially if associated with difficulties in emotion regulation, and these difficulties might be exacerbated in the context of a stressful crisis and lockdown measures, highlighting the need for intervention strategies to mitigate its negative impact.

Keywords: COVID-19, Lockdown, Eating disorders, Clinical impairment

\footnotetext{
* Correspondence: pmachado@psi.uminho.pt

${ }^{1}$ Psychotherapy and Psychopathology Research Unit - Psychology Research

Centre, University of Minho, School of Psychology, Braga, Portugal

Full list of author information is available at the end of the article
}

(c) The Author(s). 2020 Open Access This article is licensed under a Creative Commons Attribution 4.0 International License, which permits use, sharing, adaptation, distribution and reproduction in any medium or format, as long as you give appropriate credit to the original author(s) and the source, provide a link to the Creative Commons licence, and indicate if changes were made. The images or other third party material in this article are included in the article's Creative Commons licence, unless indicated otherwise in a credit line to the material. If material is not included in the article's Creative Commons licence and your intended use is not permitted by statutory regulation or exceeds the permitted use, you will need to obtain permission directly from the copyright holder. To view a copy of this licence, visit http://creativecommons.org/licenses/by/4.0/. The Creative Commons Public Domain Dedication waiver (http://creativecommons.org/publicdomain/zero/1.0/) applies to the data made available in this article, unless otherwise stated in a credit line to the data. 


\section{Plain English summary}

The current study assessed the impact of the COVID-19 pandemic crisis and the government implemented lockdown measures on a group of patients diagnosed with eating disorders, attending a specialized treatment center in Portugal. We found that those struggling with eating disorders may experience worsening of their condition, especially if they tend to have difficulties in emotion regulation, and their living routines and access to care are disrupted. This can be especially the case during stressful and disrupting events like when the state of emergency was declared in Portugal to curb the spread of COVID-19.

\section{Background}

Lockdown measures implemented by the government to prevent the coronavirus disease 2019 (COVID-19) pandemic spread resulted in marked changes in the lifestyle of the general population (e.g., closure of schools, businesses, gyms, and restaurants). During the confinement period, people were instructed to stay at home, refrain from unnecessary outings, leaving home was allowed to shop for basic needs (e.g., food, and medication), short walks around home for light exercise or walk dogs.

People with eating disorders might be disproportionally affected both by the pandemic crisis and the mitigating measures implemented to contain it [1]. For example, data, from a pilot study in Spain [2], showed that 12 out of 32 patients reported impairments in their eating disorder (ED) symptomatology, and most presented worries about increased uncertainty in their lives, being it, work, treatment, or loved ones related. Another survey [3], carried in Australia, showed that in a group of participants with ED, there was an increase in restricting, binge eating, purging, and exercise behaviors.

Rodgers and colleagues [4] proposed three pathways by which the COVID-19 pandemic might cause ED symptomatic exacerbation: 1) disruptions of daily routines, and constrains in outdoors, and social activities, that are useful in terms of emotional regulation; 2) exposure to ED-specific and anxiety-provoking media content; and 3) emotional distress caused by fears of contagion and increased health concerns. The combination of these pathways to increased risk combined with a potential lack of availability of protective factors, like social support and access to care have the potential to affect in a unique way people with eating disorders. Social distancing, lack of in-person social contact, and confinement to own home may increase negative affect, that associated with difficulties in emotional regulation might pose specific challenges to people with eating disorders.

However, results regarding the impact of lockdown in eating disorders are not consistent. For instance, most recent preliminary research [5] showed that patients with a diagnosis of Anorexia Nervosa reported significant decreases on their eating symptoms, after confinement due to COVID-19. However, these results were based on retrospective self-reported evaluation of symptoms using a novel instrument.

There is currently an urgent need for research addressing the mental health impact of the COVID-19 crisis, especially amongst those already suffering from a mental disorder [6], and an assessment of potential symptomatic mitigating or aggravating factors. The objective of the current study was to assess the impact of COVID-19 and lockdown measures on a cohort of ED patients being followed as part of an ongoing naturalistic treatment study.

\section{Methods}

\section{Participants}

Participants were recruited from an ongoing naturalistic longitudinal study conducted by our research team, focusing on treatment monitoring and follow up of patients with a diagnosis of eating disorders. In the context of that study, 99 patients aged 18 or older, currently or previously, in treatment at a Portuguese specialized hospital unit were contacted by phone in order to invite them to participate in the current survey.

Four patients refused to participate in this additional survey, and 36 weren't reachable (10 had changed their contact number, 7 had their mobile phone turned off, and 19 did not answer the call). Fifty-nine participants agreed to participate and were interviewed to obtain clinical information regarding the lockdown period. In addition, they were asked to complete a set of self-report questionnaires, of which were received 43 responses.

Of these 43 participants, 26 reported to be currently in treatment.

For the purpose of this study, we used data collected in the last available evaluation prior to COVID-19 lockdown period. Data collection regarding the impact of COVID-19 lockdown started when the end of the state of emergency, in Portugal, was announced (April 30th), and finished two weeks later (May 15th). During this data collection period, the country was under a "state of calamity" where the population had the civic duty of home confinement, in spite of the reopening of some commercial establishments and public services, and a gradual deconfinement.

\section{Measures}

Clinical interview: participants were asked about clinical information regarding the COVID-19 lockdown period, namely weight change during lockdown, current medication, if they were still under ED treatment, date of the last ED appointment, and current diagnosis. 
Eating Disorder Examination-Questionnaire (EDE-Q) $[7,8]$ : is a 28-item measure used to assess eating disorder psychopathology focusing on the last 28 days. It generates four subscales (restraint, eating concern, shape concern, and weight concern) and a global score.

Clinical Impairment Assessment (CIA) [9, 10]: is a 16item self-report questionnaire used to evaluate psychosocial impairment secondary to eating disorders, in the personal, social and cognitive domains, over the last 28 days. A higher score represents more impairment.

Impulsive Behavior Scale-Negative Urgency Subscale (UPPS-P) [11]: this subscale is composed of 12-item and is used to assess impulsivity when accompanied by negative emotions. Higher scores represent greater negative urgency.

Difficulties in Emotion Regulation Scale - Short Form (DERS-SF) [12, 13]: is an 18-item self-report questionnaire used to assess difficulties in emotion regulation. It contains six subscales: 1) Difficulties Engaging in GoalDirected Behavior, 2) Lack of Emotional Clarity, 3) Nonacceptance of Emotional Responses, 4) Limited Access to Emotion Regulation Strategies, 5) Lack of Emotional Awareness, and 6) Impulse Control Difficulties and also generate a total score. Higher scores represent greater emotion regulation difficulties.

Coronavirus Impact Scale (CIS) [14]: is an 11-item questionnaire that assesses the extent to which COVID19 pandemic changed participant's lives in the following areas: routines, family income/employment, food access, mental health care access, access to social support, experience of stress related to COVID-19 pandemic, stress/family discord, personal diagnosis of coronavirus, immediate family members diagnosed with coronavirus, and extended family members and/or close friends diagnosed with COVID-19. In the current study, we also asked participants to rate how COVID-19 pandemic changed their lives in terms of two additional areas: feeding and physical exercise. Items were answered on a 4-point Likert scale, varying from 0 ("None/No change") to 3 ("Severe"). Total score was computed by the sum of the 13 items of this adapted scale.

\section{Procedure}

In order to assess the impact of COVID-19 lockdown in this population, all participants were contacted by phone. Those who accepted to participate were interviewed by a psychologist specialized in EDs assessment and were asked to complete a set of online questionnaires, using Google Forms. Telephone interviews were conducted in a semi-structured format by 2 interviewers (APB, EL). Information regarding the aims of the study and the voluntary nature of participation were provided. This study was approved by the ethical committee of the hospital involved.

\section{Statistical analysis}

Statistical analyses were conducted using the IBM SPSS Statistics software, version 26.0. For the characterization of the sample, descriptive statistics were used.

We first assessed the impact of the lockdown period by computing change scores (CS) between post and prelockdown on the CIA total scores. A CS was considered reliable (reliable change) if exceeded the standard error of the measure.

Pearson correlations were conducted to test the association between variables under study and COVID-19 lockdown impact, and to inform the candidate variables to include in the mediation model. In order to examine the indirect effects of difficulties in emotion regulation (DERS-SF total) on the relationship between impact of COVID-19 lockdown and clinical impairment (CIA total), a regressionbased bootstrapping approach with 5000 bias-corrected resamples and 95\% confidence intervals was conducted using the PROCESS macro (v3.5) for SPSS (2020). A simple mediation model was tested in which: impact of COVID-19 lockdown was included as the independent variable $(\mathrm{X})$; CIA total was included as the dependent variable $(\mathrm{Y})$; and DERS-SF total was introduced in the model as the mediator variable $(\mathrm{M})$. The inference on mediation was based on the analysis of the indirect effects' estimates, considered significant if the interval between the lower and the upper bound of the $95 \%$ bias-corrected bootstrap confidence intervals did not contain zero $[15,16]$.

\section{Results}

\section{Participant's clinical status}

Forty-three ED patients with ages ranging from 18 to 55 years old $(M=27.60, S D=8.45)$ completed all assessments in the post confinement period. Most were women (95.3\%). Twenty had a diagnosis of anorexia nervosa, 14 of bulimia nervosa, 2 binge eating disorder, and 7 other specified feeding or eating disorder.

During the COVID-19 lockdown period, only 8 participants still went to work regularly, 6 were unemployed, 5 in layoff, 9 in prophylactic isolation, and the remaining 13 were in telework or involved in remote academic work (e.g., online classes).

Thirteen ED patients (31.0\%) reported a weight increase due to COVID-19 lockdown, 8 (19.0\%) decrease on weight, $4(9.5 \%)$ do not know their current weight, and 17 (40.5\%) report having maintained the weight during this period.

Participants' mean body mass index (BMI) based on self-reported weight and height during COVID-19 lockdown period was $21.58 \mathrm{~kg} / \mathrm{m}^{2}(\mathrm{SD}=6.85)$, significantly different from the BMI in the last assessment $20.53 \mathrm{~kg} / \mathrm{m}^{2}$ $(\mathrm{SD}=6.60)\left(\mathrm{t}_{40}=-2.26, p=.030\right)$.

Table 1 presents in detail participants' clinical and demographical data. 
Table 1 Clinical and demographical characteristics

\begin{tabular}{|c|c|c|c|c|c|}
\hline & $\mathbf{N}$ & $\%$ & $M$ & SD & Min-Max \\
\hline Age & & & 27.60 & 8.45 & $18-55$ \\
\hline \multicolumn{6}{|l|}{ Sex } \\
\hline Women & 41 & 95.3 & & & \\
\hline Men & 2 & 4.7 & & & \\
\hline \multicolumn{6}{|l|}{ Diagnosis } \\
\hline Bulimia Nervosa & 14 & 32.6 & & & \\
\hline Anorexia Nervosa & 20 & 46.5 & & & \\
\hline BED & 2 & 4.7 & & & \\
\hline OSFED & 7 & 16.3 & & & \\
\hline \multicolumn{6}{|l|}{ Currently on ED treatment } \\
\hline No & 17 & 39.5 & & & \\
\hline Yes & 26 & 60.5 & & & \\
\hline \multicolumn{6}{|l|}{ Social Lockdown } \\
\hline Yes, I'm in prophylactic isolation & 9 & 20.9 & & & \\
\hline Yes, I'm working from home & 3 & 7.0 & & & \\
\hline (telework) & 5 & 11.6 & & & \\
\hline Yes, I'm in lay off & 6 & 14.0 & & & \\
\hline Yes, I'm unemployed & 10 & 23.3 & & & \\
\hline Yes, I'm having videoconferencing & 8 & 18.6 & & & \\
\hline classes & 0 & 0.0 & & & \\
\hline No, I still go to work regularly & 2 & 4.7 & & & \\
\hline \multicolumn{6}{|l|}{ No, I still go to work sporadically } \\
\hline \multicolumn{6}{|l|}{ Other } \\
\hline Social Lockdown Period (weeks) & & & 6.71 & 1.64 & $2-9$ \\
\hline \multicolumn{6}{|l|}{ Lockdown weight change } \\
\hline Gained weight & 13 & 31.0 & & & \\
\hline Lost weight & 8 & 19.0 & & & \\
\hline Unchanged & 17 & 40.5 & & & \\
\hline Doesn't know or doesn't care & 4 & 9.5 & & & \\
\hline
\end{tabular}

Note. $N=43, B E D$ Binge Eating Disorder, OSFED Other Specified Feeding or Eating Disorder, $B M I$ Body Mass Index, ED Eating Disorders, $M$ Mean, $S D$ Standard Deviation

\section{Impact of COVID-19 lockdown}

Table 2 presents mean and standard deviation for all self-report measures in both assessment times, last available data for each participant and post-lockdown assessment. Group differences between pre-COVID-19, and post lockdown, were not significant after Bonferroni correction for multiple comparisons. Trying to extrapolate the possible impact of the lockdown period on our participants, at the individual level, we computed change scores on our measure of clinical impairment (CIA) between the previously available time point and the end of the lockdown period. Using Jacobson and Truax [17] criteria for reliable change, 16 patients improved since previously available assessment, 17 remained unchanged, and 10 deteriorated. Data showed that of the 26 patients
Table 2 Pre and post lockdown self-reported measures of eating disorder, clinical impairment, impulsivity, and difficulties in emotion regulation

\begin{tabular}{lll}
\hline & $\begin{array}{l}\text { Previous Assessment } \\
\text { M (SD) }\end{array}$ & $\begin{array}{l}\text { COVID-19 Assessment } \\
\text { M (SD) }\end{array}$ \\
\hline EDE-Q Total & $2.92(1.57)$ & $2.93(1.58)$ \\
CIA Total & $23.33(12.94)$ & $19.93(13.16)$ \\
UPPS-P & $2.59(0.66)$ & $2.65(0.75)$ \\
DERS-SF Total & $43.35(15.94)$ & $47.56(14.85)$ \\
\hline
\end{tabular}

Note. $N=43, E D E-Q$ Eating Disorder Examination-Questionnaire, UPPS-P Impulsive Behavior Scale, DERS-SF Difficulties in Emotion Regulation ScaleShort Form, CIA Clinical Impairment Assessment

currently in treatment: 8 remained unchanged (31\%), 7 deteriorated $(27 \%)$, and 11 reliably improved (42\%). Of the 17 participants not currently in treatment: 3 deteriorated (18\%), 9 remained unchanged (53\%), and 5 (29\%) improved after the lockdown period.

Results suggest that most participants, whether currently in treatment or not, considered that COVID-19 changed moderately to extremely their life in terms of routines, experiences of stress related to coronavirus pandemic, physical exercise and eating habits. The data are summarized in Table 3.

Associations between the impact of COVID-19 lockdown, eating psychopathology and psychological distress during lockdown

Table 4 shows the correlations between impact of COVID-19 lockdown, eating psychopathology and psychological distress during lockdown. Results suggest that higher impact of COVID-19 lockdown was significantly correlated with eating disorder symptoms and associated psychopathology, impulsivity, difficulties in emotion regulation and clinical impairment measured at post-lockdown. Considering the significant correlations, we tested a model including impact of COVID-19 lockdown, clinical impairment and difficulties in emotion regulation.

The mediational role of emotion regulation difficulties in the relationship between the coronavirus impact and clinical impairment secondary to ED

The total effect of the impact of COVID-19 lockdown (CIS total) on clinical impairment (CIA total) was significant, $\mathrm{c}=1.24, \mathrm{t}=4.43, p<.001$. Also, the CIS total was positively associated to difficulties in emotion regulation (DERS-SF total), $\mathrm{a}=0.97, \mathrm{CI}[.26,1.69]$, which was, in turn, positively associated with CIA total, b= 0.42 , CI $[.21, .64]$. After introducing the mediator in the model, the association between the CIS total on CIA total remained significant, $\mathrm{c}^{\prime}=0.83, \mathrm{t}=3.18, p=.003$. Finally, the indirect effect of CIS total on CIA total via DERS-SF total was positive and statistically significant, 
Table 3 Impact of COVID-19 lockdown in ED patients

\begin{tabular}{|c|c|c|c|c|c|c|}
\hline & \multicolumn{4}{|c|}{ Currently in treatment } & \multirow{2}{*}{\multicolumn{2}{|c|}{ Total }} \\
\hline & \multicolumn{2}{|l|}{ Yes } & \multicolumn{2}{|l|}{ No } & & \\
\hline & $\mathrm{N}$ & $\%$ & $\mathrm{~N}$ & $\%$ & $\mathrm{~N}$ & $\%$ \\
\hline \multicolumn{7}{|l|}{ Routines } \\
\hline No change/Mild & 6 & 23.1 & 4 & 23.5 & 10 & 23.3 \\
\hline Moderate/ Severe & 20 & 76.9 & 13 & 76.5 & 33 & 76.7 \\
\hline \multicolumn{7}{|c|}{ Family Income/Employment } \\
\hline No change/Mild & 16 & 61.5 & 16 & 94.1 & 32 & 74.4 \\
\hline Moderate/ Severe & 10 & 38.5 & 1 & 5.9 & 11 & 25.6 \\
\hline \multicolumn{7}{|l|}{ Food Access } \\
\hline No change/Mild & 26 & 100.0 & 16 & 94.1 & 42 & 97.7 \\
\hline Moderate/ Severe & 0 & 0.0 & 1 & 5.9 & 1 & 2.3 \\
\hline \multicolumn{7}{|c|}{ Medical health care access } \\
\hline No change/Mild & 15 & 57.7 & 10 & 58.8 & 25 & 58.1 \\
\hline Moderate/ Severe & 11 & 42.3 & 7 & 41.2 & 18 & 41.9 \\
\hline \multicolumn{7}{|c|}{ Mental health treatment access } \\
\hline No change/Mild & 14 & 53.8 & 13 & 76.5 & 27 & 62.8 \\
\hline Moderate/ Severe & 12 & 46.2 & 4 & 23.5 & 16 & 37.2 \\
\hline \multicolumn{7}{|c|}{ Access to extended family and non-family social supports } \\
\hline No change/Mild & 21 & 80.8 & 15 & 88.2 & 36 & 83.7 \\
\hline Moderate/ Severe & 5 & 19.2 & 2 & 11.8 & 7 & 16.3 \\
\hline \multicolumn{7}{|c|}{ Experiences of stress related to coronavirus pandemic } \\
\hline None/Mild & 11 & 42.3 & 7 & 41.2 & 18 & 41.9 \\
\hline Moderate/ Severe & 15 & 57.7 & 10 & 58.8 & 25 & 58.1 \\
\hline \multicolumn{7}{|c|}{ Stress and discord in the family } \\
\hline No change/Mild & 21 & 80.8 & 15 & 88.2 & 36 & 83.7 \\
\hline Moderate/ Severe & 5 & 19.2 & 2 & 11.8 & 7 & 16.3 \\
\hline \multicolumn{7}{|l|}{ Physical Exercise } \\
\hline No change/Mild & 10 & 38.5 & 8 & 47.1 & 18 & 41.9 \\
\hline Moderate/ Severe & 16 & 61.5 & 9 & 52.9 & 25 & 58.1 \\
\hline \multicolumn{7}{|l|}{ Feeding } \\
\hline No change/Mild & 9 & 34.6 & 8 & 47.1 & 17 & 39.5 \\
\hline Moderate/ Severe & 17 & 65.4 & 9 & 52.9 & 26 & 60.5 \\
\hline
\end{tabular}

$\mathrm{ab}=0.41$, CI $[.11, .81]$. The final model (Fig. 1), accounted for a total of $51 \%$ of the final variance of CIA total (Table 5).

\section{Discussion}

To our knowledge, this was one of the first studies to examine the impact of COVID-19 pandemic and the associated confinement measures on a cohort of patients diagnosed with eating disorders. Because these patients were participating on a naturalistic study and there was previously available data on several aspects of their psychosocial functioning, it was possible to prospectively measure the potential impact of the COVID-19 pandemic and the lockdown confinement measures.

The COVID-19 pandemic and the lockdown-imposed measures had a significant impact on patients with eating disorders. The vast majority of patients reported that the lockdown measures had impacted, moderately to severely, their life routines. Around half of the patients reported to have significant difficulties assessing medical health care and even more so accessing mental health care. Other significant changes noted were related to exercise and eating routines. This change in life routines and the increased time spent at home, in a potentially 
Table 4 Correlations between impact of COVID-19 during lockdown, eating psychopathology and psychological distress variables

\begin{tabular}{|c|c|c|c|c|c|c|}
\hline Variable & & CIS & $\mathrm{CIA}$ & EDE-Q & UPPS-P & DERS-SF \\
\hline \multirow[t]{3}{*}{ 1. CIS } & Pearson's $r$ & - & & & & \\
\hline & Upper 95\% Cl & - & & & & \\
\hline & Lower 95\% Cl & - & & & & \\
\hline \multirow[t]{3}{*}{ 2. CIA Total } & Pearson's r & $0.569^{* * *}$ & - & & & \\
\hline & Upper 95\% Cl & 0.742 & - & & & \\
\hline & Lower 95\% Cl & 0.323 & - & & & \\
\hline \multirow[t]{3}{*}{ 3. EDE-Q Total } & Pearson's r & $0.451^{* *}$ & $0.819^{* * *}$ & - & & \\
\hline & Upper 95\% Cl & 0.662 & 0.898 & - & & \\
\hline & Lower 95\% Cl & 0.175 & 0.687 & - & & \\
\hline \multirow[t]{3}{*}{ 4. UPPS-P } & Pearson's r & $0.380^{*}$ & $0.384^{*}$ & $0.392^{* *}$ & - & \\
\hline & Upper 95\% Cl & 0.611 & 0.614 & 0.620 & - & \\
\hline & Lower 95\% Cl & 0.090 & 0.095 & 0.104 & - & \\
\hline \multirow[t]{3}{*}{ 5. DERS-SF Total } & Pearson's r & $0.393^{* *}$ & $0.626^{* * *}$ & $0.505^{* * *}$ & $0.682^{* * *}$ & - \\
\hline & Upper 95\% Cl & 0.620 & 0.780 & 0.699 & 0.816 & - \\
\hline & Lower 95\% Cl & 0.106 & 0.401 & 0.241 & 0.480 & - \\
\hline
\end{tabular}

${ }^{*} p<.05,{ }^{* *} p<.01,{ }^{* * *} p<.001$

CIS Coronavirus Impact Scale, CIA Clinical Impairment Assessment, EDE-Q Eating Disorder Examination-Questionnaire, UPPS-P Impulsive Behavior Scale, DERS-SF Difficulties in Emotion Regulation Scale-Short Form

triggering environment, might have created additional challenges to ED patients.

Curiously only a minority of patients reported moderate to severe difficulty in access to extended family and non-family social support. The fact that this was a relatively young cohort of patients with easy access to social media and internet-based communication tools, and the advertised benefit of keeping in touch with social support groups (i.e., social distancing does not mean social isolation) might have contributed to this fact.

Our data suggest that patients that experience significant changes in their living routines and report highest impact of COVID-19 crisis and lockdown measures, also experience increased psychological distress, which, in

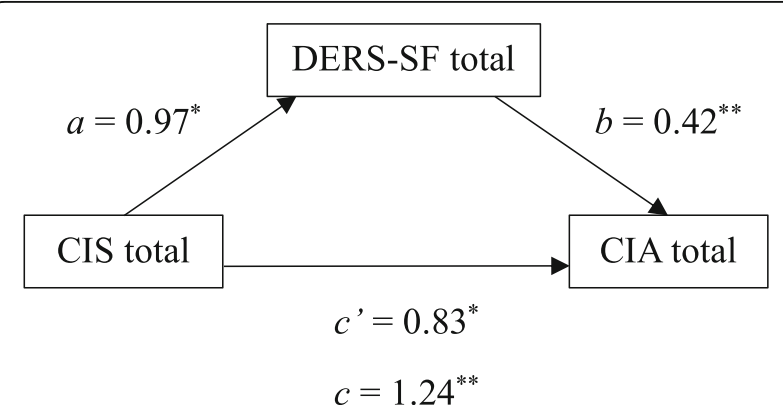

Fig. 1 Statistical diagram of the simple mediation model of the relationship between the coronavirus impact and clinical impairment, mediated by emotion regulation difficulties. CIS Coronavirus Impact Scale; DERS-SF - Difficulties in Emotion Regulation Scale-Short Form; CIA - Clinical Impairment Assessment. * $p<.05,{ }^{* *} p<.01$ turn, may result in more disordered eating and clinical impairment. The changes imposed by the COVID-19 lockdown were significantly associated with clinical impairment. Most importantly, this impact was mediated by emotion regulation difficulties.

Although the COVID-19 crisis had impacted the general population as well, there are reasons to believe that those struggling with a mental health problem, and transient difficulties in accessing regular mental health care services, might be disproportionally affected by the pandemic crisis and associated measures [18]. However, further research that includes control group comparisons are needed to understand whether ED patients are significantly more affected than the general population.

To the best of our knowledge, this is one of the first studies to assess the impact of COVID-19 and lockdown measures in a cohort of ED patients that is not completely retrospective. The timing of the data collection, and the availability of data from pre-COVID-19 provided a unique opportunity to assess the impact of the pandemic crisis and associated lockdown measures. However, the current study has limitations, namely: 1) the use of a convenient, relatively small, sample based on an ongoing naturalistic study, 2) the use of phone interviews in the second wave of data collection as opposed to face-to-face in the first one; 3) the use of self-report measures and self-report height and weight that might have introduced some bias; 4) most patients that we approached, for various reasons, did not take part of the study; and, 5) findings reflect the experience of a sample of patients in Portugal. 
Table 5 Model coefficients for the mediation model

\begin{tabular}{|c|c|c|c|c|c|c|c|c|}
\hline \multirow[b]{3}{*}{ Antecedent } & \multicolumn{8}{|c|}{ Consequent } \\
\hline & & \multicolumn{3}{|c|}{$M$ (DERS-SF total) } & & \multicolumn{3}{|c|}{$Y(\mathrm{CIA}$ total $)$} \\
\hline & & Coeff. & $S E$ & $p$ & & Coeff. & $S E$ & $p$ \\
\hline $\mathrm{X}$ (CIS total) & $a$ & 0.97 & 0.35 & .009 & $c^{\prime}$ & 0.83 & 0.26 & .003 \\
\hline M (DERS-SF total) & & - & - & - & $b$ & 0.42 & 0.11 & $<.001$ \\
\hline \multirow[t]{3}{*}{ Constant } & $i_{M}$ & 35.07 & 5.02 & $<.001$ & $i_{Y}$ & -10.85 & 5.05 & .038 \\
\hline & & \multicolumn{3}{|c|}{$R^{2}=0.15$} & & \multicolumn{2}{|c|}{$R^{2}=0.51$} & \\
\hline & & \multicolumn{3}{|c|}{$F(1,41)=7.51, p=.009$} & & \multicolumn{2}{|c|}{$F(2,40)=21.20, p=<.001$} & \\
\hline
\end{tabular}

Note. $\mathrm{N}=43, \mathrm{CIS}$ Coronavirus Impact Scale, DERS-SF Difficulties in Emotion Regulation Scale - Short Form, CIA Clinical Impairment Assessment

\section{Conclusions}

Those struggling with eating disorders may experience worsening of their condition, especially if associated with difficulties in emotion regulation, and these difficulties might be exacerbated in the context of a stressful crisis and lockdown measures. Given the need to plan for future adjustments to deal with COVID-19 is important to take these findings into consideration. Future interventions need to include self-help strategies that provide structure when usual routines are disrupted and adaptive emotion regulation strategies to deal with stressful events.

\section{Abbreviations}

ED: Eating Disorders; COVID-19: Coronavirus disease 2019;i EDE-Q: Eating Disorder Examination-Questionnaire; CIA: Clinical Impairment Assessment; UPPS-P: Impulsive Behavior Scale-Negative Urgency Subscale; DERSSF: Difficulties in Emotion Regulation Scale - Short Form; CIS: Coronavirus Impact Scale; CS: Change scores; SPSS: Statistical package for the social sciences

\section{Acknowledgements}

The authors wish to acknowledge, Sertório Timóteo, MD and Patrícia Nunes, MD, at the University Hospital Center of São João, for their collaboration in collecting data for this study. Also, we would like to thank the participants for their willingness to contribute to this study.

\section{Authors' contributions}

All authors contributed to the planning and design of the study. PPM took primary responsibility for the manuscript, including reviewing relevant literature and drafting the paper for publication. APB was responsible for recruitment of participants and data collection, contributed to its analysis and interpretation. RR managed the database, contributed to its analysis, and interpretation. TFR contributed to its analysis and interpretation. EL collaborated in data collection and participants recruiting. IB conducted diagnostic interviews. All authors assisted with literature review and editing of the manuscript. All authors read and approved the final manuscript.

\section{Funding}

This work was conducted at the Psychology Research Centre (PSI/01662), School of Psychology, University of Minho, and partially supported by the Portuguese Foundation for Science and Technology and the Portuguese Ministry of Science, Technology and Higher Education (UID/PSI/01662/2019), through the national funds (PIDDAC); and a grant by the Portuguese Foundation for Science and Technology and the Portuguese Ministry of Science, Technology and Higher Education through national funds and cofinanced by FEDER through COMPETE2020 under the PT2020 Partnership Agreement (POCl-01-0145-FEDER-028145) to P. Machado (PI).

\section{Availability of data and materials}

The datasets used and/or analysed during the current study are available from the corresponding author on reasonable request.
Ethics approval and consent to participate

The Ethics Committee of the University Hospital Centre of São João approved the study, and informed consent was obtained from all participants.

Consent for publication

Not applicable.

\section{Competing interests}

The authors declare that they have no competing interests.

\section{Author details}

${ }^{1}$ Psychotherapy and Psychopathology Research Unit - Psychology Research Centre, University of Minho, School of Psychology, Braga, Portugal. ${ }^{2}$ Faculty of Medicine, Psychiatry Department, University Hospital Center of São João, 4200-319 Porto, Portugal.

Received: 2 August 2020 Accepted: 14 October 2020

Published online: 02 November 2020

References

1. Touyz S, Lacey H, Hay P. Eating disorders in the time of COVID-19. J Eat Disord. 2020:8:8-10. https://doi.org/10.1186/s40337-020-00295-3..

2. Fernández-Aranda F, Casas M, Claes L, Bryan DC, Favaro A, Granero R, et al. COVID-19 and implications for eating disorders. Eur Eat Disord Rev. 2020;28: 239-45. https://doi.org/10.1002/erv.2738.

3. Phillipou A, Meyer D, Neill E, Tan EJ, Toh WL, Van Rheenen TE, et al. Eating and exercise behaviors in eating disorders and the general population during the COVID-19 pandemic in Australia: initial results from the COLLATE project. Int. J. Eat. Disord. 2020;53:1158-65. https:// doi.org/10.1002/eat.23317.

4. Rodgers RF, Lombardo C, Cerolini S, Franko DL, Omori M, FullerTyszkiewicz M, et al. The impact of the COVID-19 pandemic on eating disorder risk and symptoms. Int J Eat Disord. 2020;53:1166-70. https:// doi.org/10.1002/eat.23318

5. Fernández-Aranda F, Munguía L, Mestre-Bach G, et al. COVID Isolation Eating Scale (CIES): Analysis of the impact of confinement in eating disorders and obesity - A collaborative international study. Eur Eat Disord Rev. n/a(n/a). https://doi.org/10.1002/erv.2784.

6. Holmes EA, O'Connor RC, Perry VH, Tracey I, Wessely S, Arseneault L, et al. Multidisciplinary research priorities for the COVID-19 pandemic: a call for action for mental health science. Lancet Psychiatry. 2020;7:547-60. https:// doi.org/10.1016/S2215-0366(20)30168-11.

7. Fairburn CG, Beglin SJ. Eating Disorder Examination Questionnaire (6.0). In: Fairburn CG, editor. Cogn. Behav. Ther. Eat. Disord. New York: Guilford Press; 2008. p. 309-13.

8. Machado P, Martins C, Vaz A, Conceição E, Pinto-Bastos A, Gonçalves S. Eating disorder examination questionnaire (EDE-Q): psychometric properties and norms for the Portuguese population. Eur Eat Disord Rev. 2014;22:44853. https://doi.org/10.1002/erv.2318.

9. Bohn K, Fairburn CG. The Clinical Impairment Assessment Questionnaire (CIA 3.0). In: Fairburn CG, editor. Cogn. Behav. Ther. Eat. Disord. New York, NY: Guilford Press; 2008. p. 315-8.

10. Vaz AR, Conceição E, Pinto-Bastos A, Silva D, Machado PPP. Validation of the Portuguese version of the clinical impairment assessment (CIA) in eating 
disorders' patients. Eat Weight Disord. 2020;25:627-35. https://doi.org/10. 1007/s40519-019-00661-4.

11. Whiteside SP, Lynam DR, Miller JD, Reynolds SK. Validation of the UPPS impulsive behavior scale: a four factor model of impulsivity. Eur J Personal. 2005:574:559-74. https://doi.org/10.1002/per.556.

12. Kaufman EA, Xia M, Fosco G, Yaptangco M, Skidmore CR, Crowell SE. The difficulties in emotion regulation scale short form (DERS-SF): validation and replication in adolescent and adult samples. J Psychopathol Behav Assess. 2016;38:443-55. https://doi.org/10.1007/s10862-015-9529-3.

13. Moreira H, Gouveia MJ, Canavarro MC. A bifactor analysis of the difficulties in emotion regulation scale - short form (DERS-SF) in a sample of adolescents and adults. Curr Psychol Current Psychol. 2020. https://doi.org/ 10.1007/s12144-019-00602-5.

14. Kaufman J, Stoddard J. Coronavirus impact scale. 2020; https://disasterinfo. nlm.nih.gov/search/?source=2587. Accessed 28 April 2020.

15. Hayes AF, Rockwood NJ. Regression-based statistical mediation and moderation analysis in clinical research: observations, recommendations, and implementation. Behav Res Ther. 2017;98:39-57. https://doi.org/10. 1016/j.brat.2016.11.001

16. Hayes AF. Introduction to mediation, moderation, and conditional process analysis: A regression-based approach. 2nd ed: New York: Guilford Press; 2018.

17. Jacobson NS, Truax P. Clinical significance: a statistical approach to defining meaningful change in psychotherapy research. J Consult Clin Psychol. 1991; 59:12-9. https://doi.org/10.1037/10109-042.

18. Moreno C, Wykes T, Galderisi S, et al. How mental health care should change as a consequence of the COVID-19 pandemic. Lancet Psychiatry. 2020;7(9):813-24. https://doi.org/10.1016/\$2215-0366(20)30307-2.

\section{Publisher's Note}

Springer Nature remains neutral with regard to jurisdictional claims in published maps and institutional affiliations.

Ready to submit your research? Choose BMC and benefit from:

- fast, convenient online submission

- thorough peer review by experienced researchers in your field

- rapid publication on acceptance

- support for research data, including large and complex data types

- gold Open Access which fosters wider collaboration and increased citations

- maximum visibility for your research: over $100 \mathrm{M}$ website views per year

At $\mathrm{BMC}$, research is always in progress.

Learn more biomedcentral.com/submissions 